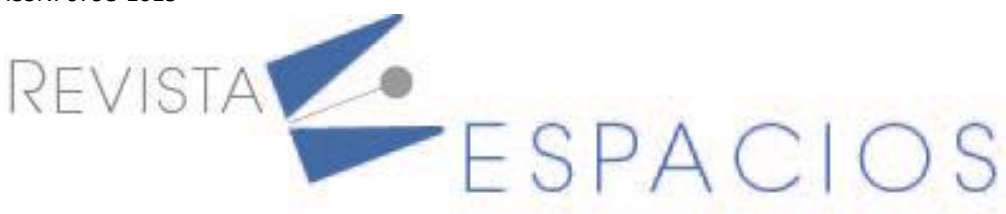

Vol. 41 (47) $2020 \cdot$ Art. 9

\title{
Estructura de celosía o lattice aplicada a filtros adaptativos en el procesamiento digital de señales
}

\section{Lattice structure applied to adaptive filters in digital signal processing.}

RIVERA, María F. ${ }^{1}$

\begin{abstract}
Resumen
La estructura Lattice por su modularidad permite canalizar datos, ofrece propiedades deseables de estabilidad, rápida convergencia y seguimiento (tracking). Otras propiedades como el cascadeo de secciones idénticas, coeficientes con magnitudes inferiores a la unidad, prueba de estabilidad por inspección, buenas características de eficiencia y robustez en los cálculos de los errores de redondeo numérico resultan atractivas para aplicaciones de filtrado adaptativo dado que la solución recursiva de la estimación de los mínimos cuadrados produce naturalmente un filtro de estructura Lattice.

Palabras clave: filtros adaptativos, estructura en celosía o lattice, filtro feedback y procesado digital de señales.

Abstract

The lattice structure for the great modularity allows us to canalize the information, offers desirable properties of stability, excellent numerical precision, rapid convergence, and fast-tracking. Other properties such as cascading of identical sections, coefficients less than unity magnitudes, test due to inspection, good features of efficiency, and robustness in the calculations of numerical rounding errors are attractive for applications of adaptive filtering since the recursive solution of the least-squares estimation filter naturally Lattice structure. key words: adaptive filters, lattice structure, feedback filter and digital signal processing.
\end{abstract}

\section{Introducción}

En el campo del procesamiento digital de señales es frecuente utilizar estructuras de filtro que pueden modelar y analizar la señal digital con base en sus características o en la retroalimentación de su salida. Este tipo de estructuras de filtro ofrece ventajas especialmente para efectos de la cuantificación. Como caso de particular importancia se destacan las denominadas estructuras de filtrado adaptable en cascada, en paralelo y en celosía.

Así mismo, se entiende por filtros adaptativos, aquellas estructuras ajustables que tienen la capacidad de cambiar su respuesta automáticamente para mejorar su desempeño, es identificado igualmente como aquel dispositivo autodiseñable con base en un algoritmo recursivo que permite al filtro desempeñarse satisfactoriamente en un ambiente en el cual no se dispone de un conocimiento amplio de las características relevantes de la señal, es decir, el algoritmo parte de algunas condiciones iniciales predeterminadas, que evidencian completa ignorancia del ambiente. Tales aplicaciones incluyen: la ecualización del canal, la cancelación de ruido y el modelado de sistemas entre otras, así mismo, se emplean filtros con coeficientes

${ }^{1}$ Docente-Investigador. Departamento Electrónica . Universidad de Investigación y Desarrollo - UDI. mrivera7@udi.edu.co 
ajustables y que son los propiamente identificados como filtros adaptativos por incorporar algoritmos que permiten adaptar los coeficientes del filtro a los parámetros estadísticos de la señal. (Rivera, 2013)

La estructura de filtros Lattice es una alternativa para realizar una función de transferencia de un filtro digital que no tiene un número mínimo de multiplicadores y sumadores para la realización de una función de transferencia pero si ofrece muchas propiedades ventajosas como por ejemplo cascadeo de secciones idénticas, coeficientes con magnitudes menores a la unidad, prueba de estabilidad por inspección, buenas características de redondeo numérico y es particularmente útil para el filtrado adaptable dado que la solución recursiva de la estimación de los mínimos cuadrados produce naturalmente un filtro de estructura Lattice. Así mismo se caracteriza porque ortogonaliza la señal de entrada en una base de etapa a etapa permitiendo capacidades de rápida convergencia y seguimiento. No obstante que muchas técnicas alternativas han sido desarrolladas para estimar los coeficientes de reflexión que parametrizan la estructura Lattice, el método de los mínimos cuadrados recursivos actualiza la estimación de los mínimos cuadrados a través de la observación de cada muestra. Este procedimiento conlleva una estimación optima y requiere únicamente un costo computacional ligeramente mayor al de otras técnicas. (Galindo, 2008)

Las técnicas de estimación adaptables modifican los parámetros estimados del filtro de acuerdo a los datos observados actualizados, para cada nueva muestra, la estimación recursiva utilizando el filtro Lattice genera nuevos coeficientes de reflexión y errores de predicción para cada orden del filtro. Cambiar cada coeficiente del filtro para cada nueva muestra resulta importante en las aplicaciones donde la rápida convergencia o el seguimiento (tracking) de señales altamente variables son necesarias. Sin embargo, para aplicaciones donde la dinámica es lenta los resultados de observar la señal por cierto tiempo resultan importantes. (Cowan y Grant, 1986) Así entonces, la estructura de Celosía o Lattice, está motivada por la teoría del modelo auto-regresivo de la señal.

\section{Características de la estructura de celosía o lattice}

Es una estructura modular fundamentada en un bloque denominado cruz o escalera el cual se va añadiendo uno a uno dependiendo del orden del filtro (figura 1). Dicha estructura está constituida por bloques y cada bloque consta de un retardo $\left(z^{-1}\right)$ unidos a través de líneas horizontales, puntos de suma, líneas cruzadas de coeficientes, denominados coeficientes de reflexión $\left(k_{m}\right)$, los cuales son estimados en cada muestra con el fin de minimizar el error de predicción de adelanto $f_{0}(n)$ y de atraso $g_{0}(n)$ como se muestra en la figura 2.

Figura 1

Estructura Lattice de $M$ etapas

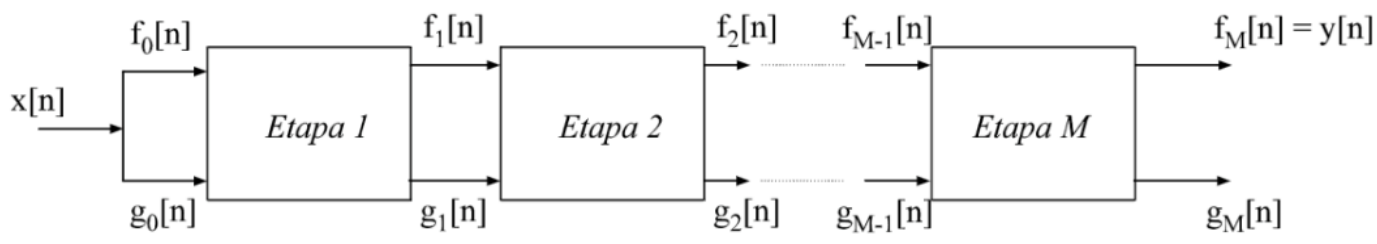

Fuente : Apollo y Cordova, 2010 
Figura 2

Estructura Lattice de filtro FIR de segundo orden

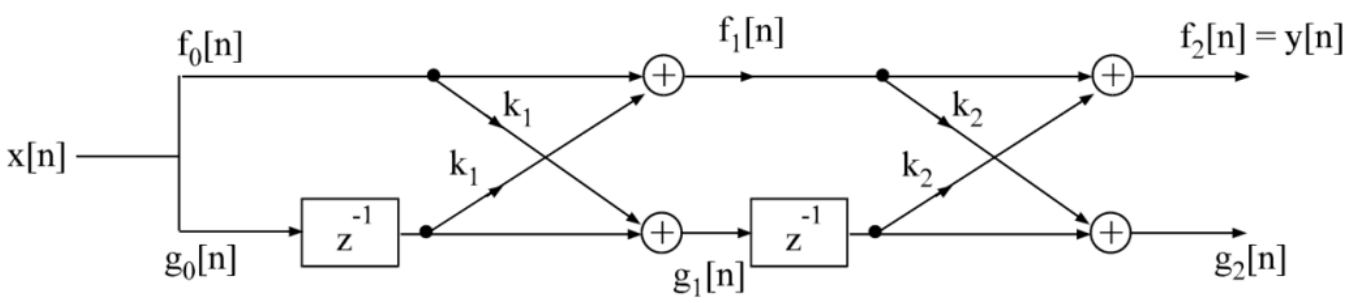

Fuente: Apolo y Córdova, 2010

De esta forma se observa que las ecuaciones recursivas que describen la estructura Lattice son:

$$
\begin{gathered}
f_{m}(n+1)=f_{m-1}(n+1)+K_{m}(n+1) g_{m-1}(n) \\
g_{m}(n+1)=g_{m-1}(n)+K_{m}(n+1) f_{m-1}(n+1) \\
f_{0}(n)=g_{0}(n)=x(n)
\end{gathered}
$$

Donde $f_{m}(n+1)$ y $g_{m}(n+1)$ son las secuencias del error de predicción de adelanto y atraso respectivamente, $K_{m}(n+1)$ es el coeficiente de reflexión y $m=1,2, \ldots, M$ según el orden del filtro.

La estructura lattice, presenta una serie de propiedades de gran interés para aplicaciones de filtrado adaptativo. La Tabla 1 ilustra de manera comparativa dichas propiedades con las correspondientes a los filtros FIR e IIR.

Tabla 1

\begin{tabular}{|c|c|c|c|}
\hline & IIR & FIR & LATTICE \\
\hline $\begin{array}{l}\text { USOS Y } \\
\text { APLICACIONES }\end{array}$ & Equivalentes a filtros análogos. & $\begin{array}{l}\text { Transmisión de datos. } \\
\text { Audio digital. } \\
\text { Procesamiento de imágenes. }\end{array}$ & $\begin{array}{l}\text { Procesamiento digital de } \\
\text { señales de voz. } \\
\text { Implementación de Filtros } \\
\text { adaptativos. } \\
\text { Tratamiento de señales } \\
\text { geofísicas. }\end{array}$ \\
\hline VENTAJAS & $\begin{array}{l}\text { Memoria infinita } \\
\text { En un mismo diseño de filtro, la } \\
\text { longitud del filtro IIR más corta } \\
\text { que en FIR. } \\
\text { Mejor atenuación que los FIR. } \\
\text { Pueden proporcionar pendientes } \\
\text { de corte muy pronunciadas. }\end{array}$ & $\begin{array}{l}\text { Estructura del filtro siempre es } \\
\text { estable. } \\
\text { Diseño para tener respuesta en } \\
\text { fase lineal. } \\
\text { No entran en oscilación debido } \\
\text { a que no tienen realimentación. }\end{array}$ & $\begin{array}{l}\text { Número reducido de } \\
\text { coeficientes permite que } \\
\text { grandes bloques de datos } \\
\text { puedan ser modelados en } \\
\text { tiempo real. }\end{array}$ \\
\hline DESVENTAJAS & $\begin{array}{l}\text { Producen en general distorsión } \\
\text { de fase, es decir la fase no es } \\
\text { lineal con la frecuencia. } \\
\text { Transitorio que no se limita a un } \\
\text { intervalo de tiempo finito. }\end{array}$ & $\begin{array}{l}\text { Para especificaciones dadas } \\
\text { requieren un filtro de orden } \\
\text { mucho mayor que los filtros IIR. } \\
\text { Memoria finita. } \\
\text { Número de operaciones y } \\
\text { tiempo de procesamiento altos } \\
\text { comparados con los de IIR. }\end{array}$ & $\begin{array}{l}\text { El filtro de estructura celosía } \\
\text { puede ser FIR o IIR por tanto } \\
\text { también posee sus } \\
\text { desventajas. }\end{array}$ \\
\hline
\end{tabular}

Propiedades y otras características comparativas de la arquitectura de celosía o lattice

Fuente: Propia 
De la tabla 1, se nota que la estructura Lattice es adecuada para la implementación de filtros, es así que se mostrara la adecuacion de la estructura Lattice a los filtros FIR ( sistema todo cero, MA) filtros IIR (sistema todo polos, AR) y combinación de los dos anteriores (sistema ceros y polos, ARMA) de la siguiente forma:

\subsection{Predictor lineal de adelanto}

Adecuación de la estructura Lattice a un filtro FIR , sistema todo ceros, (Moving Average, MA).

Partiendo de una funcion de transferencia $H(z)=\sum_{k=0}^{M} h(k) z^{-k}$ se define un conjunto de filtros

$$
\begin{gathered}
A_{m}(z)=\sum_{k=0}^{m} \alpha_{m}(k) z^{-k} \quad m \geq 1 ; \quad \alpha_{m}(0)=1 \\
H(z)=A_{m}(z)
\end{gathered}
$$

Luego la respuesta al impulso es $h_{m}(0)=1, \ldots, h_{m}(k)=\alpha_{m}(k)$

Haciendo el paso al dominio del tiempo se tiene

$$
Y(z)=A_{m}(z) X(z) \Rightarrow y(n)=\underbrace{x(n)+\sum_{k=1}^{m} \alpha_{m}(k) x(n-k)}_{\text {Predictor lineal de adelanto }}
$$

Para un filtro de orden $\mathrm{m}=1, y(n)=x(n)+\alpha_{1}(1) x(n-1)$

Haciendo uso de las ecuaciones (1) y (2) para un filtro de orden m=1 estas quedarían así:

$$
\left.\begin{array}{c}
f_{1}(n)=y(n)=f_{0}(n)+k_{1} g_{0}(n-1) \\
g_{1}(n)=f_{0}(n) k_{1}+g_{0}(n-1)
\end{array}\right\} \Rightarrow y(n)=x(n)+k_{1} x(n-1)
$$

Luego $k_{1}=\alpha_{1}(1)$ coeficiente para filtro de primer orden.

Si se aumenta el orden del filtro se obtendría:

$$
\begin{aligned}
& \left.\begin{array}{c}
f_{2}(n)=y(n)= \\
f_{1}(n)+k_{2} g_{1}(n-1) \\
g_{2}(n)=f_{1}(n) k_{2}+g_{1}(n-1)
\end{array}\right\} \\
& \Rightarrow \begin{array}{c}
y(n)=\left[f_{0}(n)+k_{1} g_{0}(n-1)\right]+k_{2}\left[f_{0}(n-1) k_{1}+g_{0}(n-2)\right] \\
y(n)=x(n)+k_{1}\left(1+k_{2}\right) x(n-1)+k_{2} x(n-2)
\end{array}
\end{aligned}
$$

Los coeficientes del filtro serían:

$$
\alpha_{2}(2)=k_{2}, \quad \alpha_{1}(1)=k_{1}\left(1+k_{2}\right)
$$

al calcular $g_{2}(n)=k_{2} x(n)+k_{1}\left(1+k_{2}\right) x(n-1)+x(n-2)$ se puede notar que la ecuacion es muy parecida a $f_{2}(n)$ reflejando los coeficientes.

Es así que de forma general para un sistema de varias etapas como se ve en la figura 1, la salida sería: 


$$
\begin{gathered}
y(n)=f_{M}(n) \\
f_{m}(n)=\sum_{k=0}^{m} \alpha_{m}(k) x(n-k)
\end{gathered}
$$

De la misma forma para $g_{m}(n)$

$$
\begin{gathered}
g_{m}(n)=\sum_{k=0}^{m} \beta_{m}(k) x(n-k) \\
\beta_{m}(k)=\alpha_{m}(\mathrm{~m}-\mathrm{k})
\end{gathered}
$$

Se puede deducir $B_{m}(z)=z^{-m} A_{m}\left(z^{-1}\right)$

Para la obtención de los coeficientes de reflexión a partir de la función de transferencia de debe aplicar la transformada $z$ a las ecuaciones generales de la estructura Lattice así:

$$
\begin{aligned}
& f_{o}(n)=x(n) \quad F_{0}(z)=X(z) \\
& g_{0}(n)=x(n) \quad \mathrm{G}_{0}(\mathrm{z})=\mathrm{X}(\mathrm{z}) \\
& f_{m}(n)=f_{m-1}(\mathrm{n})+\mathrm{k}_{m} \mathrm{~g}_{m-1}(\mathrm{n}-1) \quad \stackrel{T Z}{\rightarrow} \quad \mathrm{F}_{m}(\mathrm{z})=\mathrm{F}_{m-1}(\mathrm{z})+\mathrm{k}_{m} \mathrm{z}^{-1} G_{m-1}(\mathrm{z}) \\
& g_{m}(n)=k_{m} f_{m-1}(\mathrm{n})+\mathrm{g}_{m-1}(\mathrm{n}-1) \quad \mathrm{G}_{m}(\mathrm{z})=\mathrm{k}_{m} \mathrm{G}_{m-1}(\mathrm{z})+\mathrm{z}^{-1} G_{m-1}(\mathrm{z})
\end{aligned}
$$

Ya que $F_{m}(z)=A_{m}(z) X(z) \quad$ y $\quad G_{m}(z)=B_{m}(z) X(z)$, se pueden obtener los coeficientes de reflexión a partir de la ecuacion de transferencia haciendo la siguiente relación:

$$
\begin{gathered}
A_{m-1}(z)=\frac{A_{m}(z)-k_{m} B_{m}(z)}{1-k_{m}^{2}} \\
\text { Como } \quad B_{m}(z)=z^{-m} A_{m}\left(z^{-1}\right), \quad \alpha_{m}(m)=k_{m} \quad \alpha_{m}(0)=1 .
\end{gathered}
$$

En sentido contrario, obtener la función de transferencia a partir de los coeficientes de reflexíón se relaciona de la siguiente forma:

$$
\begin{gathered}
A_{m}(z)=A_{m-1}(z)+k_{m} z^{-1} B_{m-1}(z) \\
B_{m}(z)=z^{-m} A_{m}\left(z^{-1}\right) \\
B_{0}(z)=A_{0}(z)=1
\end{gathered}
$$

\subsection{Predictor lineal de atraso}

Adecuación de la estructura Lattice a un filtro IIR, sistema todo polos, (Auto Regressive, AR).

Para un sistema todo polos su función de transferencia sería:

$$
H(z)=\frac{1}{1+\sum_{k=1}^{N} \alpha_{N}(k) z^{-k}}=\frac{1}{A_{N}(z)}
$$

La ecuación de salida para un filtro IIR en el dominio del tiempo sería de la siguiente forma: 


$$
y(n)=\underbrace{-\sum_{k=1}^{N} \alpha_{N}(k) y(n-k)+x(n)}_{\text {Predictor lineal de atraso }}
$$

Como ya se conocen las ecuaciones para un sistema FIR adecuando la estructura Lattice y haciendo un intercambio de la entrada y la salida se obtendrian las ecuaciones para un sistema IIR en Lattice todo polos de la siguiente forma:

$$
\begin{gathered}
x(n)=f_{N}(n) \\
f_{m-1}(n)=f_{m}(\mathrm{n})-\mathrm{k}_{m} \mathrm{~g}_{m-1}(\mathrm{n}-1) \\
g_{m}(\mathrm{n})=\mathrm{k}_{m} \mathrm{f}_{m-1}(\mathrm{n})+\mathrm{g}_{m-1}(\mathrm{n}-1) \\
\mathrm{y}(\mathrm{n})=\mathrm{f}_{0}(\mathrm{n})=\mathrm{g}_{0}(\mathrm{n})
\end{gathered}
$$

Al hacer los cambios mencionados los diagramas de bloques de forma general seria el siguiente,

Figura 3

Estructura Lattice de filtro IIR

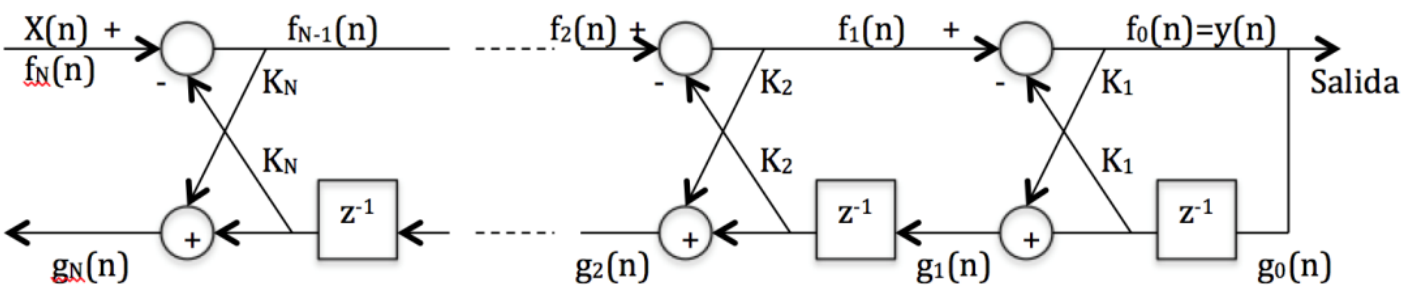

Fuente: Proakis, 2007

En la figura anterior se puede observar la realimentación a través de la señal $g(n)$ caracteristica de un sistema recursivo. Los coeficientes de reflexión se obtienen de igual manera que al aplicar un filtro FIR.

Ahora se considera un sistema ARMA (AutoRegressive Moving Average models), sistema con ceros y polos.

$$
H(z)=\frac{\sum_{k=0}^{M} c_{M}(k) z^{-k}}{1+\sum_{k=1}^{N} a_{N} z^{-k}}=\frac{C_{M}(z)}{A_{N}(z)} \quad M<N
$$

Se podría usar una variable intermedia lo que permite obtener:

$$
H(z)=\frac{Y(z)}{W(z)} \frac{W(z)}{X(z)}=\frac{C_{M}(z)}{A_{N}(z)} \rightarrow \begin{aligned}
\frac{1}{A_{N}(z)} & =\frac{W(z)}{X(z)} \\
C_{M}(z) & =\frac{Y(z)}{W(z)}
\end{aligned}
$$

Las ecuaciones en el dominio del tiempo serían: 


$$
\begin{aligned}
w(n) & =-\sum_{k=1}^{N} a_{N}(k) w(n-k)+x(n) \leftarrow \text { IIR todo polos } \\
y(n) & =\sum_{k=0}^{M} c_{M}(k) w(n-k) \quad \leftarrow \text { Combinación lineal de la salida del sistema todo polos }
\end{aligned}
$$

De la figura 3 se puede observar que $g(n)$ es una combinación lineal de la salida actual y anteriores, luego,

$$
\frac{G_{m}(z)}{Y(z)}=B_{m}(z)
$$

y se considera,

$$
y(n)=\sum_{m=0}^{M} v_{m} g_{m}(n)
$$

Como,

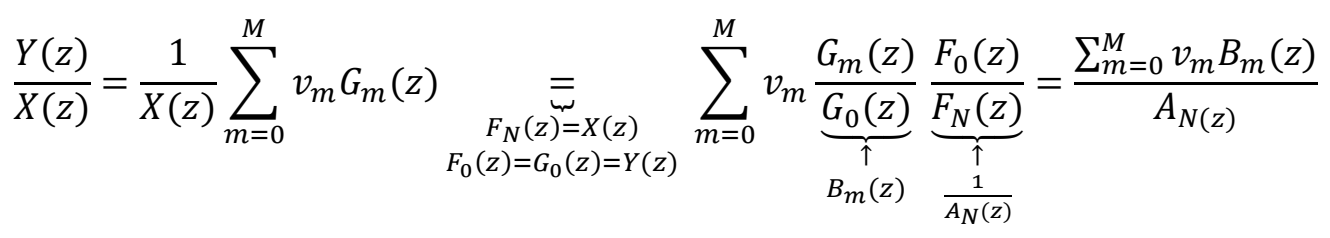

Comparando (33) con (32) ecuacion general de un sistema ARMA se obtiene,

$C_{M}(z)=\sum_{m=0}^{M} v_{m} B_{m}(z)$ que se puede expresar de forma recursiva así $C_{m-1}(z)=C_{M}(z)-v_{m} B_{m}(z)$ siendo $v_{m}=c_{m}(m)$

\section{Aplicación del algoritmo LMS, NLMS y ARY en la estructura lattice}

Un método para la estimación de los coeficientes de reflexión (Fujii et al, 2007), aplicando los métodos convencionales de algoritmos adaptativos, resulta de la aplicación de la estructura lattice al algoritmo LMS y NLMS, para el efecto los coeficientes de reflexión se calculan como coeficientes de correlación cruzada entre los predictores de adelanto y atraso en cada etapa del filtro lattice adaptativo.

La configuración del filtro lattice mostrada en la figura 2, se puede expresar de forma general como:

$$
y(n)=f_{m}(n)=f_{0}(n)-\sum_{m=1}^{M} K_{m}(n) g_{m-1}(n-1)
$$

En donde $f_{m}(n)$ es la salida del filtro lattice con sus componentes $f_{0}(n)$ que es un valor de predicción y la sumatoria de los coeficientes de reflexión $K_{m}(n)$ por el error de predicción de atraso $g_{m-1}(n-1)$. El segundo componente de la ecuación (23) se puede considerar como un filtro FIR de error de predicción como se ilustro en las secciones anteriores. Con base en las metodologías utilizadas en los tratamientos de los filtros FIR en lo relativo a la estimación de coeficientes, para los filtros lattice el procedimiento resulta similar.

Así mismo, al minimizar el error de predicción de adelanto final se obtendrían los coeficientes de reflexión usando la siguiente estimación al hacer uso del algoritmo LMS: 


$$
\begin{gathered}
\boldsymbol{K}_{\boldsymbol{m}}(\boldsymbol{n}+\mathbf{1})=\boldsymbol{K}_{\boldsymbol{m}}(\boldsymbol{n})+\boldsymbol{\mu}\left[\boldsymbol{f}_{\boldsymbol{m}}(\boldsymbol{n}) \boldsymbol{g}_{\boldsymbol{m}-\mathbf{1}}(\boldsymbol{n}-\mathbf{1})+\boldsymbol{g}_{\boldsymbol{m}}(\boldsymbol{n}) \boldsymbol{f}_{\boldsymbol{m}-\mathbf{1}}(\boldsymbol{n})\right], \quad \mathbf{0}<\boldsymbol{m} \leq \boldsymbol{M} \\
e_{0}(n)=d(n)-g_{0}(n) w_{0}(n) \\
e_{m}(n)=e_{m-1}(n)-g_{m}(n) w_{m}(n) \\
w_{m}(n+1)=w_{m}(n)+\mu e_{m}(n) g_{m}(n)
\end{gathered}
$$

El método de Burg (Haykin, 2002) garantiza que los coeficientes de reflexión son menores a la unidad.

Así mismo para el algoritmo NLMS la ecuación que me permite obtener los coeficientes de reflexión es la siguiente (Qi, 2008):

$$
K_{m}(n+1)=K_{m}(n)+\mu \frac{f_{m}(n) g_{m-1}(n-1}{\sum_{i=1}^{M}\left(g_{m-1}(i-1)\right)^{2}}, \quad 0<m \leq M
$$

De esta misma forma se hace uso de las ecuaciones que describes el filtro $A R \gamma$ y al adecuarlas a la estructura Lattice se obtiene como resultado:

$$
\begin{gathered}
y(n)=x(n) w(n) \\
e(n)=y(n)-d(n) \\
g(n)=\frac{e(n)+\gamma x^{T}(n) q_{1}(n-1)}{1+\alpha \gamma x^{T}(n) m_{1} x(n)} \\
q_{1}(n)=\frac{\gamma}{\alpha+\gamma}\left[q_{1}(n-1)-\alpha g(n) m_{1} x(n)\right]
\end{gathered}
$$

De donde los coeficientes de reflexión son:

$$
K_{m}(n)=K_{m}(n-1)+\alpha q_{1}(n)
$$

\section{Simulación}

El filtro Lattice adaptativo es usado generalmente para sistemas de predicción lineal y es aplicado a un sistema de identificación que permitirá modelar un filtro adaptativo de un sistema desconocido. Del cual ademas se observara el comportamiento de filtros convencionales como LMS y NLMS además del filtro ARY al adecuarlos a la estructura Lattice.

El sistema de identificación utilizado se caracteriza por un filtro FIR de respuesta impulsiva $h$, señal de entrada $x(n)$ es ruido blanco gaussiano con media cero y $\sigma^{2}=0.005$. Los parámetros a idntificar se suponen invariantes en el tiempo, dados por $y(n)=-2 u(n)+2 u(n-1)$, orden del filtro $M=2$ siendo igual al número de etapas de la estructura lattice, número de muestras $N=5000$ y número de experiencias $S=100$.

En las figuras 5 a 7 se aprecian las señales de error, coeficientes propios del filtro y coeficientes de reflección para los filtros LMS, NLMS y ARy aplicando la estructura Lattice. 
Figura 5

Señales filtro LMS-Lattice.
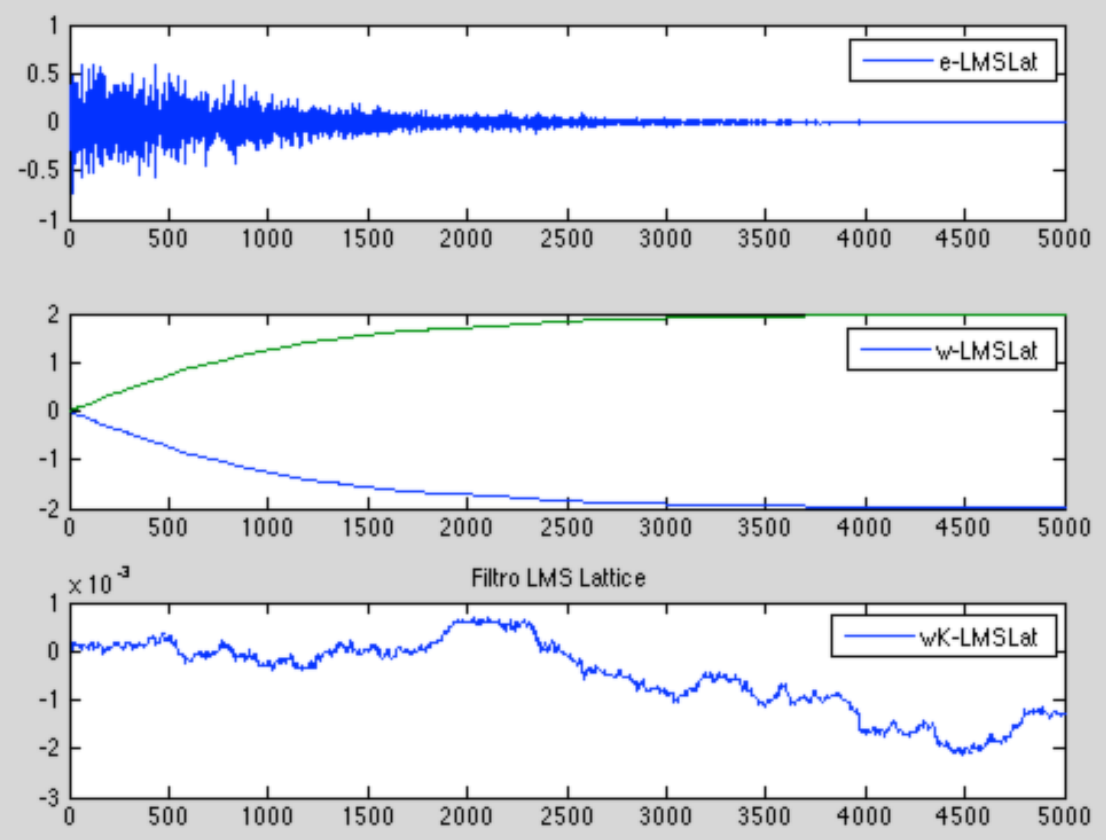

Fuente: Propia

Figura 6

Señales filtro NLMS-Lattice
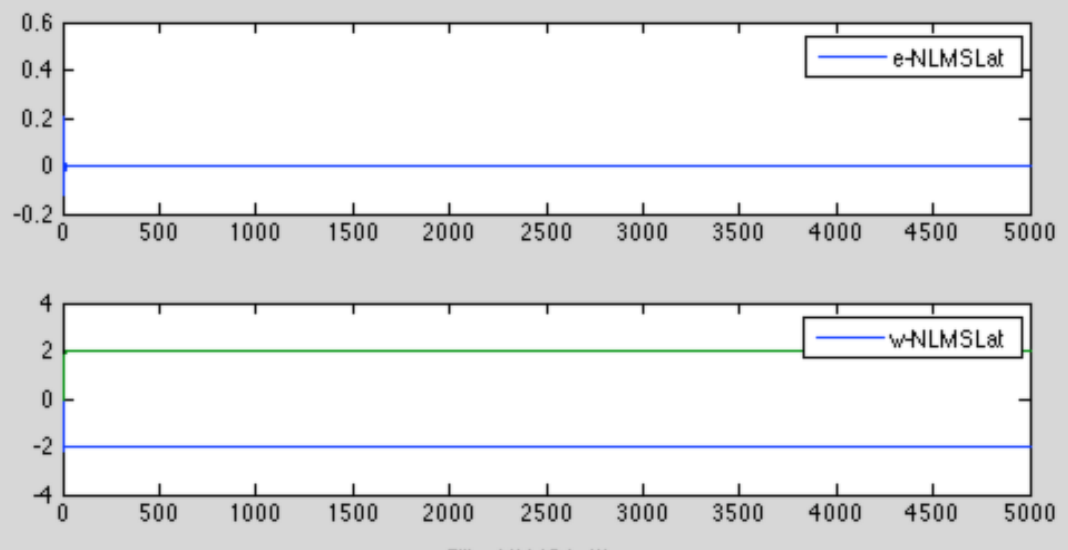

Filtro NLMS Lattice

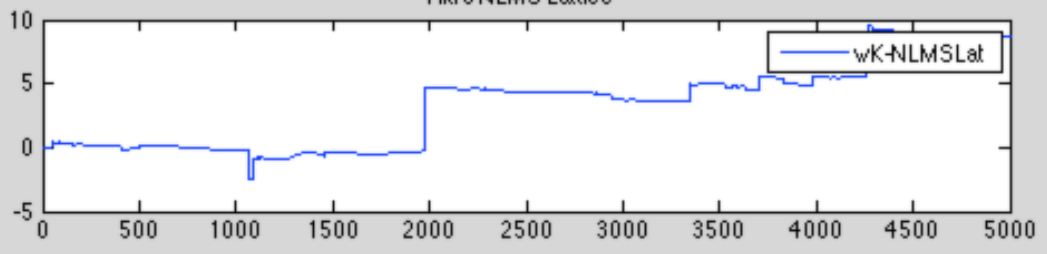

Fuente: Propia 
Figura 7

Señales filtro ARY-Lattice.
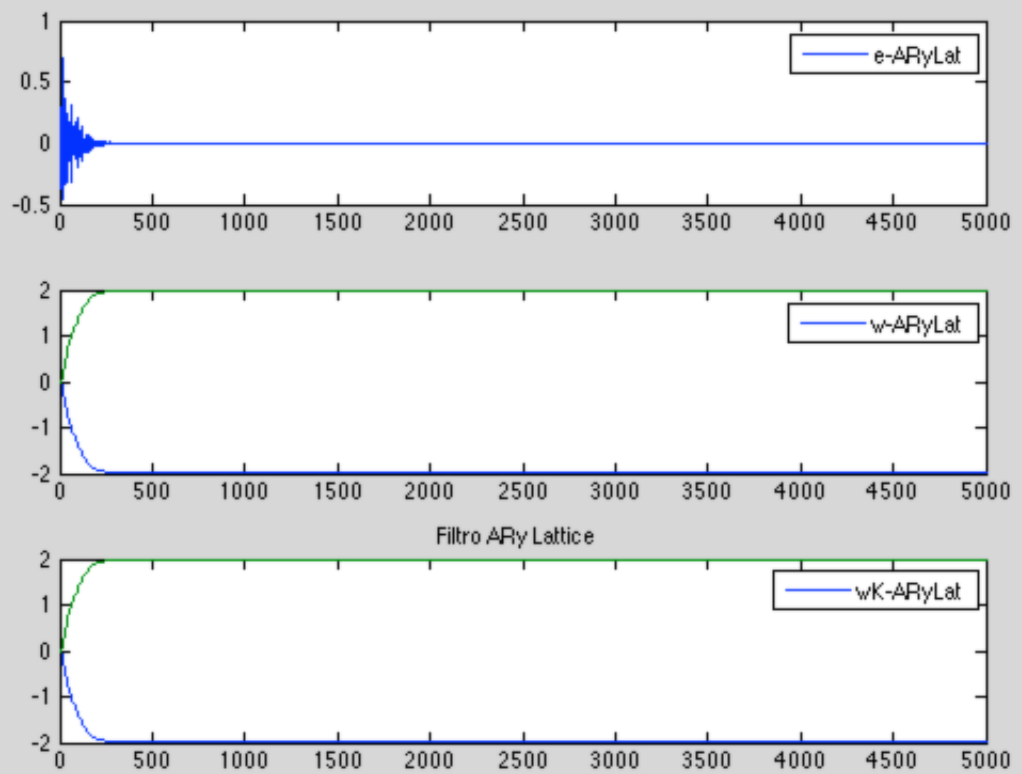

Fuente: Propia

En las Figura 8 se puede observar el comportamiento del error cuadrático medio para los filtros LMS, NLMS y $\mathrm{AR} \gamma$ tanto en su forma convencional como al aplicar la estructura lattice.

Figura 8

Error Cuadratico Medio para los filtros LMS, LMS- Lattice, NLMS, NLMS- Lattice, ARy y ARy-Lattice.

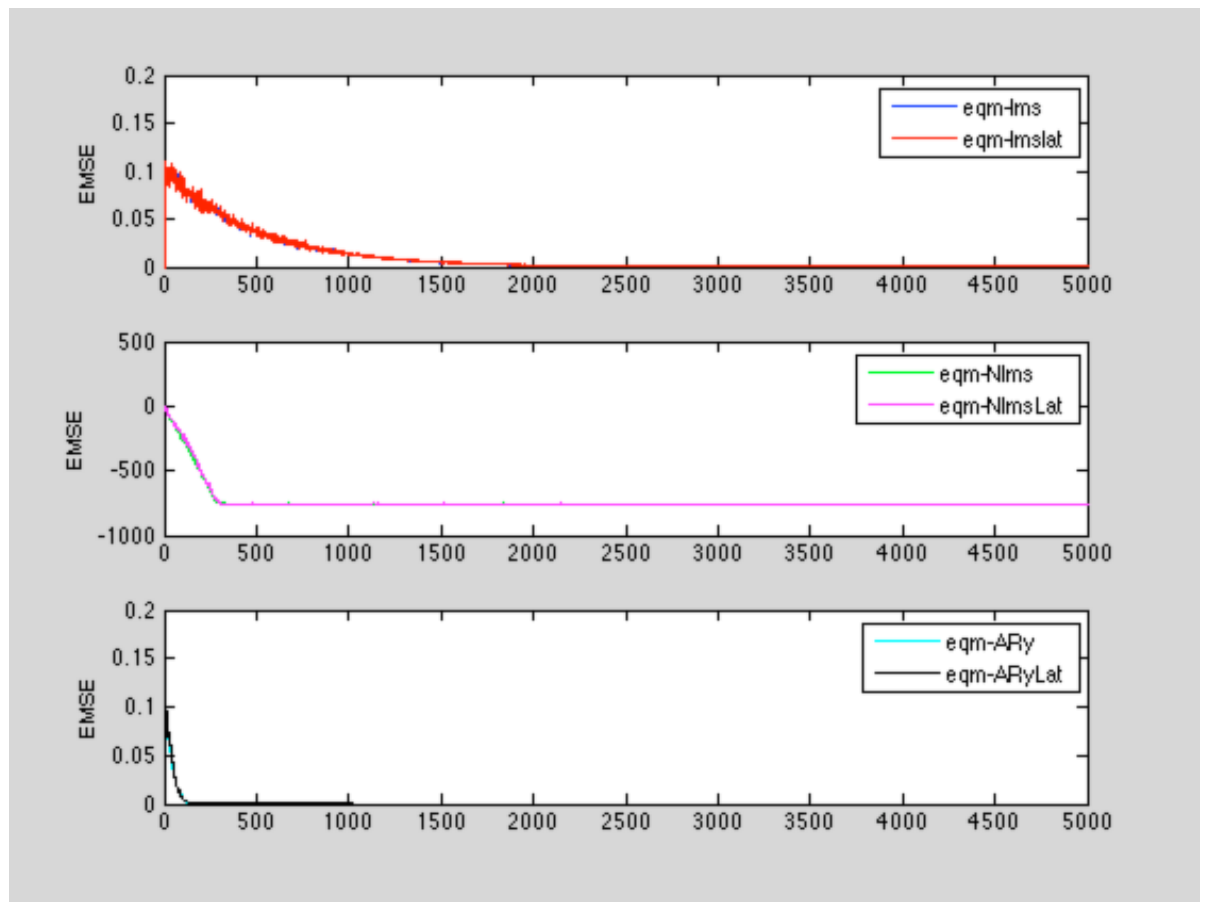

Fuente: Propia 
La Figura 9 permite apreciar el comportamiento de los filtros LMS, NLMS y AR $\gamma$ al adecuarlos a la estructua Lattice y verificar el comportamiento de los predictores de adelanto y atraso.

Figura 9

Predictor de adelanto y atraso filtros LMS-Lattice, NLMS-Lattice y ARY-Lattice

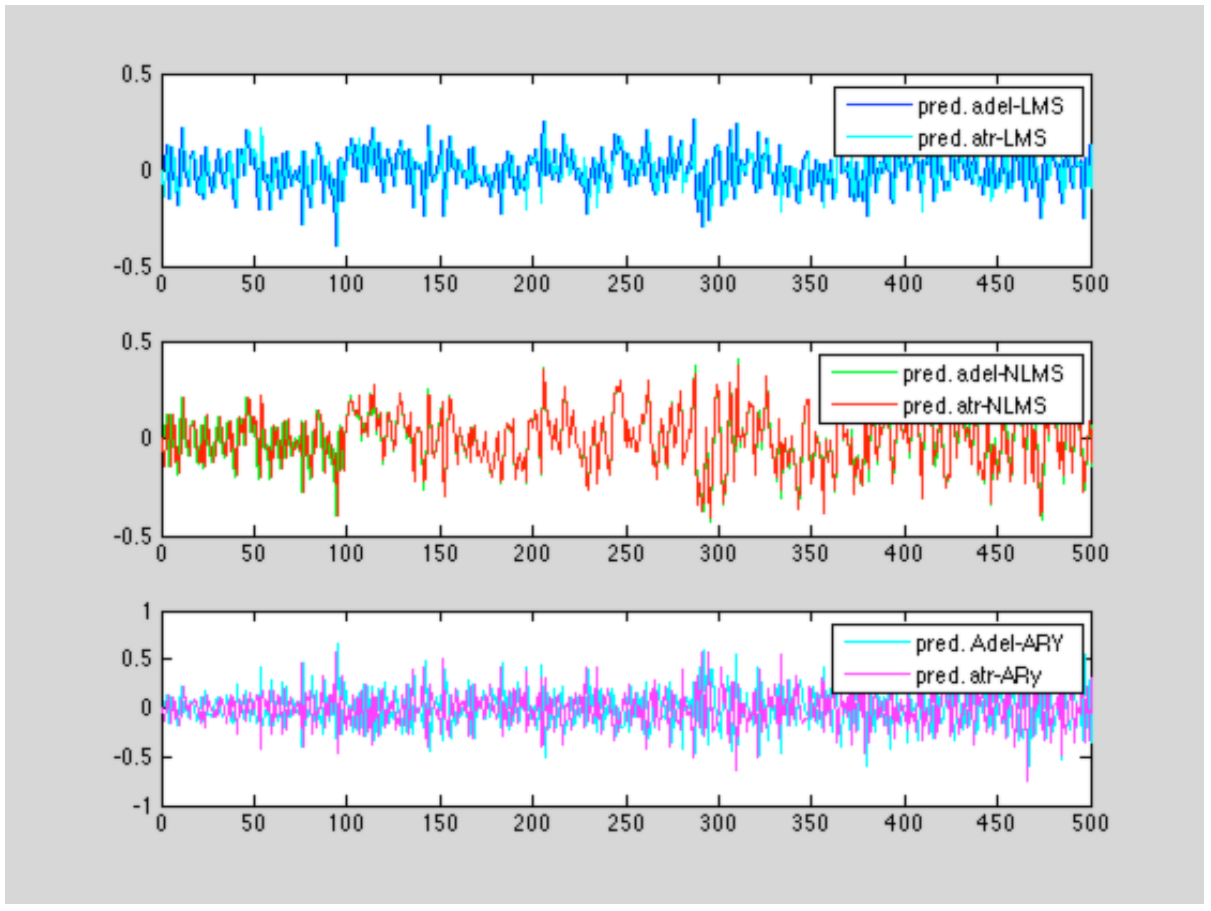

Fuente: Propia

En las tablas 2, 3 y 4 se realiza el registro del parametro $m u \_k$ propio de la estructura lattice y los diferentes valores del error cuadratico medio para los diferentes filtros LMS, NLMS y ARY al adecuarlos a la estructura lattice. Es así como se puede evidenciar que el mejor comportamiento lo ofrece el filtro NLMS-Lattice ya que ofrece el menor valor de error cuadrático medio, al variar el parametro $\mathrm{M}$ propio del algoritmo ARy.

Tabla 2

Error cuadrático medio de los filtros LMS, NLMS y ARp en estructura Lattice para diferentes valores del paso de adaptación Lattice (mu_k) y orden del filtro $\mathrm{M}=2$.

\begin{tabular}{ccccc}
\hline mu_k & Eqm_LMS- Lattice & Eqm_NLMS- Lattice & Eqm_ARY-Lattice \\
\hline 0.001 & 0.0067 & $2.9841 \mathrm{e}-29$ & $2.7702 \mathrm{e}-05$ \\
0.005 & 0.0068 & $3.2099 \mathrm{e}-29$ & $2.9303 \mathrm{e}-05$ \\
0.01 & 0.0067 & $6.2459 \mathrm{e}-29$ & $2.4906 \mathrm{e}-05$ \\
0.05 & 0.0067 & $2.7096 \mathrm{e}-28$ & $2.4266 \mathrm{e}-05$ \\
$\mathrm{M}=2$ & 0.1 & 0.0067 & $8.3752 \mathrm{e}-30$ & $2.5071 \mathrm{e}-05$ \\
& 0.5 & 0.0068 & $1.0303 \mathrm{e}-29$ & $2.7158 \mathrm{e}-05$ \\
& 1 & 0.0067 & $1.0329 \mathrm{e}-29$ & $2.6404 \mathrm{e}-05$ \\
2 & 0.0068 & $9.0558 \mathrm{e}-31$ & $2.7599 \mathrm{e}-05$ \\
5 & 0.0067 & $1.0130 \mathrm{e}-27$ & $2.6637 \mathrm{e}-05$ \\
& 10 & 0.0068 & $1.5884 \mathrm{e}-28$ & $2.7760 \mathrm{e}-05$ \\
& 30 & 0.0068 & $3.7675 \mathrm{e}-29$ & $2.7026 \mathrm{e}-05$ \\
50 & 0.0068 & $3.3025 \mathrm{e}-30$ & $2.8105 \mathrm{e}-05$ \\
\hline
\end{tabular}

Fuente : Propia 
Tabla 3

Error cuadratico medio de los filtros LMS, NLMS y ARy en estructura Lattice para diferentes valores del paso de adaptación Lattice (mu_k) y orden del filtro $\mathrm{M}=4$.

\begin{tabular}{ccccc}
\hline & mu_k & Eqm_LMS- Lattice & Eqm_NLMS- Lattice & Eqm_ARY-Lattice \\
\cline { 2 - 5 } $\mathbf{M}=\mathbf{0}$ & 0.001 & 0.0041 & $1.5692 \mathrm{e}-33$ & $3.3858 \mathrm{e}-11$ \\
0.005 & 0.0042 & $1.5738 \mathrm{e}-33$ & $3.7445 \mathrm{e}-11$ \\
0.01 & 0.0042 & $1.5790 \mathrm{e}-33$ & $3.6576 \mathrm{e}-11$ \\
& 0.05 & 0.0041 & $1.5706 \mathrm{e}-33$ & $2.9504 \mathrm{e}-11$ \\
& 0.1 & 0.0041 & $1.5635 \mathrm{e}-33$ & $3.2727 \mathrm{e}-11$ \\
& 0.5 & 0.0042 & $1.5714 \mathrm{e}-33$ & $3.3514 \mathrm{e}-11$ \\
1 & 0.0041 & $1.5719 \mathrm{e}-33$ & $3.4032 \mathrm{e}-11$ \\
& 2 & 0.0042 & $1.5748 \mathrm{e}-33$ & $3.8148 \mathrm{e}-11$ \\
& 5 & 0.0041 & $1.5711 \mathrm{e}-33$ & $3.4023 \mathrm{e}-11$ \\
& 10 & 0.0041 & $1.5723 \mathrm{e}-33$ & $3.4040 \mathrm{e}-11$ \\
30 & 0.0041 & $1.5655 \mathrm{e}-33$ & $2.9697 \mathrm{e}-11$ \\
50 & 0.0041 & $1.5650 \mathrm{e}-33$ & $2.9738 \mathrm{e}-11$ \\
\hline
\end{tabular}

Fuente: Propia

Tabla 4

Error cuadratico medio de los filtros LMS, NLMS y ARy en estructura Lattice para diferentes valores del paso de adaptación Lattice (mu_k) y orden del filtro $\mathrm{M}=6$

\begin{tabular}{ccccc}
\hline & mu_k & Eqm_LMS- Lattice & Eqm_NLMS- Lattice & Eqm_ARY-Lattice \\
\cline { 2 - 5 } $\mathbf{0 . 0 0 1}$ & 0.0117 & $7.3138 \mathrm{e}-33$ & $2.1977 \mathrm{e}-10$ \\
$\mathbf{M}=6$ & 0.0116 & $7.3462 \mathrm{e}-33$ & $2.1438 \mathrm{e}-10$ \\
0.01 & 0.0116 & $7.3268 \mathrm{e}-33$ & $2.2927 \mathrm{e}-10$ \\
& 0.05 & 0.0116 & $7.3381 \mathrm{e}-33$ & $1.9913 \mathrm{e}-10$ \\
& 0.1 & 0.0116 & $7.3347 \mathrm{e}-33$ & $1.9083 \mathrm{e}-10$ \\
& 0.5 & 0.0116 & $7.3337 \mathrm{e}-33$ & $2.1398 \mathrm{e}-10$ \\
1 & 0.0117 & $7.3344 \mathrm{e}-33$ & $2.6679 \mathrm{e}-10$ \\
2 & 0.0118 & $7.3392 \mathrm{e}-33$ & $2.9102 \mathrm{e}-10$ \\
& 5 & 0.0116 & $7.3332 \mathrm{e}-33$ & $2.4262 \mathrm{e}-10$ \\
10 & 0.0116 & $7.3650 \mathrm{e}-33$ & $2.4848 \mathrm{e}-10$ \\
30 & 0.0116 & $7.3256 \mathrm{e}-33$ & $2.0026 \mathrm{e}-10$ \\
& 50 & 0.0116 & $7.3197 \mathrm{e}-33$ & $1.9383 \mathrm{e}-10$ \\
\hline
\end{tabular}

Fuente: Propia

\section{Conclusiones}

Los filtros digitales constituyen una de las herramientas utilizadas en el procesamiento de señales y su clasificación se realiza con base en la estructura o arquitectura que la caracteriza. Para la implementación de filtros adaptativos es frecuente el uso denominado realización en celosía o estructura Lattice, en la que los parámetros en celosía, o coeficientes de reflexión, están relacionados con los coeficientes del filtro de la estructura FIR (finite-duration-impulse-response) en la forma directa. 
Así mismo, permite convertir los coeficientes del filtro FIR en los coeficientes de reflexión y viceversa. Los algoritmos de filtrado adaptativo en los que la estructura del filtro es una celosía, también denominada Lattice, tienen como fundamento el método de mínimos cuadrados, ofrecen diversas propiedades deseables, incluyendo la eficiencia y robustez en los cálculos de los errores de redondeo, ventajas que hacen al filtro en celosía o Lattice muy atractivo para aplicaciones de filtrado adaptativo.

Los filtros adaptativos por su naturaleza constituyen sistemas autodiseñables que son también autoajustables a diferentes ambientes, razón por la que tienen aplicación en diversos campos como control, comunicaciones, procesamiento de señales de radar o sonar, cancelación de interferencias, control activo de ruido, ingeniería biomédica, etc.

La característica común de estas aplicaciones con esquema de filtrado adaptable, es que todas involucran un proceso de filtrado de algún tipo de señal para coincidir con una respuesta deseada. Los parámetros del filtro son actualizados obteniendo un conjunto de medidas de las señales subyacentes para ser aplicadas al filtro adaptativo de manera tal que, la diferencia entre la salida del filtro y la respuesta deseada sea minimizada ya sea en un sentido estadístico o determinístico. Así entonces, son reconocidas aplicaciones de: modelado, modelado inverso, predicción lineal y cancelación de interferencias.

\section{Referencias bibliográficas}

Apolo, H. N. y Córdova, A. E. (2010). Modelación matemática y simulación de un filtro digital hibrido FIR adaptativo lineal óptimo. Tesis. Universidad Politécnica Salesiana, Cuenca, Ecuador.

Alvarez, J., Cuez, M., y Vargas., P. (2011). Implementaciones en Matlab de los Algoritmos Adaptativos para los Sistemas de Antenas Inteligentes. Escuela Superior Politecnica del Litoral ESPOL. Julio. Ecuador

Ayala, I, L. (1982). On a New Adaptive Lattice Algorithm for Recursive Filters. IEEE Transactions on Acoustics, speech, and signal processing, Vol. ASSP-30, No. 2, Abril.

Akaike, H. (1974). A new look at the statistical model identification. IEEE transactions on automatic control. Vol 19, 716-723.

Berberidis, K y Theodoridis, S. (1990). A Normalized Lattice Algorithm for AR Apectral Analysis and System Identifacation Filters with Symmetric Impulse Response. IEEE Transactions on Acoustics, speech, and signal processing. Vol. 38. No 3. Marzo.

Calderón, A. K y Mejía, J. A. (2012) Sintetizador Digital para las Notas de un Violin con Base en Filtros Celosía. Tesis de Grado, Instituto Politecnico Nacional, Escuela Superior de Ingeniería Mecánica y Eléctrica, Marzo, Mexico D. F.

Chakraborty, M y Mitra, A. (2004). The Gradient Adaptive Lattice Algorithm in Block Floating Point Format. IEEE ICASSP. Pp. 849-852.

Choi, J. H., Cho, H., Jeong, J. J., y Kim, S. W. (2013) Combination of step sizes for Affine Projection Algorithm with Variable Mixing Parameter. Electronics Letters 29th, Vol 49, No 18, Agosto.

Chun, S. H., Kim, Y. H., Choi, K. J., Kim, J. Y., Kim J. H., Kim, D., y Hahn, C. K. (2010) Adaptive Digital Predistortions base don Affine Projection Algorithm for WCDMA power amplifier Applications. IEEE.

Cifuentes, V., larussi, E y Clausse, A. (2012) Metodo de Lattice-Boltzmann para segmentación de texturas de imágenes. Asociación Argentina de Mecanica Computacional. Vol 31. Pags. 3027-3036. Argentina. Noviembre 
Cowan, C. F. N. and Grant, P. M. (1986) Adaptive Filters. Prentice - Hall.

De, P y Howard Fan, H (1999) Stable Lattice Filters and Their Continuous-Time Limits. IEEE Transactions on Circuits and Systems-II: Analog and Digital Signal Processing, Vol 46, No 2, Febrero.

Dedieu, H y Chételat, O. (1993) Automatic derivation of adaptive algorithm for a large class of filter structures. IEEE. Suiza.

Della Vecchia, G (2003) Una Estructura en Cascada FIR para Predicción Lineal Adaptativa. Presentación Power Ponit. Septiembre.

Dubin, Dale. (2000). Rapid Interpretation of EKG's. Cover Publishing, 6 ed. ISBN 978-0912912066.

Fujii, K., Tanaka, M., Sasaoka, N. y Itoh, Y. (2007). Method estimating reflection coefficients of adaptive lattice filter and its application to system identifacation. Acoust. Sci. \& Tech. Vol. 28. Págs. 98-104. Febrero.

Haykin, S, (2002). Adaptive Filter Theory, 4ed, Upper Saddle River, Prentice Hall, New Jersey.

Galindo, H. (2008) Estudio e implementación de algoritmos de filtrado adaptable en aritmetica de punto fijo. Tesis de Maestría, universidad Nacional Autonoma de Mexico, Mexico.

Ketchum, J. y Proakis, J. (1982). Adaptive Algorithms for Estimating and Suppressing Narrow-Band Interference in PN Spread-Spectrum Systems. IEEE Trans. Communications, Vol. COM-30, págs. 913-923, Mayo.

Nichols, H. y Proakis, J. (1977). MLD and MSE Algorithms for Adaptive Detection of Digital Signals in the Presence of Interchannel Interference. IEEE Trans. Information Theory, Vol. IT-23, págs. 563-575, septiembre.

Picinbono, B. y Skwirzynski, J. K. (1978). Adaptive Signal Processing for Detection and Communication. En Communication Systems and Random Process Theory, Paises Bajos.

Proakis, J. G. y Manolakis, D. G. (2007) Tratamiento Digital de Señales, 4ed, Pearson Education S.A. Madrid.

Qi, H. (2008) Analysis and Application of Gradient Adaptive Lattice Filtering Algorithm. International Conference on Computer and Electrical Engineering. IEEE.

Rivera. M. F. (2013).Efecto de la configuración Affine en el Algoritmo Acelerador versión y (AR Y). Tesis de Maestría, Universidad del Cauca, Popayan, Colombia.

Windrow, B., Mantey, P. y Griffiths, L. (1967). Adaptative Antenna systems. Proc. IEEE, Vol. 55, págs. 21432159, diciembre.

Esta obra está bajo una Licencia Creative Commons Attribución-NoCommercial 4.0 International

(cc) BY-NC 